\title{
Front Matter: Volume 7925
}

, "Front Matter: Volume 7925," Proc. SPIE 7925, Frontiers in Ultrafast Optics: Biomedical, Scientific, and Industrial Applications XI, 792501 (28 March 2011); doi: $10.1117 / 12.891453$

SPIE. Event: SPIE LASE, 2011, San Francisco, California, United States 


\title{
PROCEEDINGS OF SPIE
}

\section{Frontiers in Ultrafast Optics: Biomedical, Scientific, and Industrial Applications XI}

\author{
Alexander Heisterkamp \\ Joseph Neev \\ Stefan Nolte \\ Editors \\ 23-26 January 2011 \\ San Francisco, California, United States \\ Sponsored by \\ SPIE \\ Cosponsored by \\ APE GmbH (Germany) \\ Amplitude Systèmes (France) \\ Time-Bandwidth Products AG (Switzerland) \\ Published by \\ SPIE
}

Volume 7925 
The papers included in this volume were part of the technical conference cited on the cover and title page. Papers were selected and subject to review by the editors and conference program committee. Some conference presentations may not be available for publication. The papers published in these proceedings reflect the work and thoughts of the authors and are published herein as submitted. The publisher is not responsible for the validity of the information or for any outcomes resulting from reliance thereon.

Please use the following format to cite material from this book:

Author(s), "Title of Paper," in Frontiers in Ultrafast Optics: Biomedical, Scientific, and Industrial Applications XI, edited by Alexander Heisterkamp, Joseph Neev, Stefan Nolte, Proceedings of SPIE Vol. 7925 (SPIE, Bellingham, WA, 2011) Article CID Number.

ISSN 0277-786X

ISBN 9780819484628

Published by

SPIE

P.O. Box 10, Bellingham, Washington 98227-0010 USA

Telephone +1 3606763290 (Pacific Time) · Fax +1 3606471445

SPIE.org

Copyright (@ 2011, Society of Photo-Optical Instrumentation Engineers

Copying of material in this book for internal or personal use, or for the internal or personal use of specific clients, beyond the fair use provisions granted by the U.S. Copyright Law is authorized by SPIE subject to payment of copying fees. The Transactional Reporting Service base fee for this volume is $\$ 18.00$ per article (or portion thereof), which should be paid directly to the Copyright Clearance Center (CCC), 222 Rosewood Drive, Danvers, MA 01923. Payment may also be made electronically through CCC Online at copyright.com. Other copying for republication, resale, advertising or promotion, or any form of systematic or multiple reproduction of any material in this book is prohibited except with permission in writing from the publisher. The CCC fee code is 0277-786X/11/\$18.00.

Printed in the United States of America.

Publication of record for individual papers is online in the SPIE Digital Library.

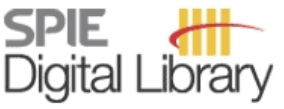

SPIEDigitalLibrary.org

Paper Numbering: Proceedings of SPIE follow an e-First publication model, with papers published first online and then in print and on CD-ROM. Papers are published as they are submitted and meet publication criteria. A unique, consistent, permanent citation identifier (CID) number is assigned to each article at the time of the first publication. Utilization of CIDs allows articles to be fully citable as soon as they are published online, and connects the same identifier to all online, print, and electronic versions of the publication. SPIE uses a six-digit CID article numbering system in which:

- The first four digits correspond to the SPIE volume number.

- The last two digits indicate publication order within the volume using a Base 36 numbering system employing both numerals and letters. These two-number sets start with 00, 01, 02, 03, 04 , 05, 06, 07, 08, 09, OA, OB ... 0Z, followed by 10-1Z, 20-2Z, etc.

The CID number appears on each page of the manuscript. The complete citation is used on the first page, and an abbreviated version on subsequent pages. Numbers in the index correspond to the last two digits of the six-digit CID number. 


\section{Contents}

ix Conference Committee

xi Ultrasensitive phase measurement with spatial spectral interferometry in an enhancement cavity [7925-310] (Abstract Only)

X. Gu, I. Pupeza, T. Udem, E. Fill, J. Kaster, Max-Planck-Institut für Quantenoptik (Germany);

T. Eidam, J. Limpert, A. Tünnerman, Friedrich-Schiller-Univ. Jena (Germany); F. Krausz, Max-Planck-Institut für Quantenoptik (Germany)

xv Microfabrication by optical tweezers (Plenary Paper) (7921-102)

R. Ghadiri, T. Weigel, C. Esen, A. Ostendorf, Ruhr-Univ. Bochum (Germany)

\section{SESSION 1 BIOMEDICAL APPLICATIONS I: IMAGING AND SENSING}

792502 Microcavity biosensing (Invited Paper) [7925-01]

F. Vollmer, Max Planck Institute for the Science of Light (Germany)

792503 Ultrafast laser induced condensation of molecules [7925-02]

L. GU, S. K. Mohanty, The Univ. of Texas at Arlington (United States)

792504 Method for imaging quantum dots during exposure to gamma radiation [7925-03]

A. N. Immucci, A. Chamson-Reig, Univ. of Western Ontario (Canada); K. Yu, National Research Council Canada (Canada); D. Wilkinson, Defence Research and Development Canada (Canada); C. Li, Health Canada (Canada); R. Z. Stodilka, J. J. L. Carson, Univ. of Western Ontario (Canada)

792505 Femtosecond single optical fiber tweezers enabled two-photon fluorescence excitation of trapped microscopic objects [7925-04]

Y. N. Mishra, The Univ. of Texas at Arlington (United States) and Cochin Univ. of Science \& Technology (India); M. Pinto, N. Ingle, S. K. Mohanty, The Univ. of Texas at Arlington (United States)

\section{SESSION 2 BIOMEDICAL APPLICATIONS II}

792506 Femtosecond laser patterning of biological materials (Invited Paper) [7925-05]

C. P. Grigoropoulos, H. Jeon, H. Hidai, D. J. Hwang, Univ. of California, Berkeley (United States)

792507 Generation of UV and blue light by using off-axis pumping for fluorescence lifetime spectroscopy [7925-06]

S. Taccheo, Swansea Univ. (United Kingdom); C. D'Andrea, A. Bassi, R. Cubeddu, Politecnico di Milano (Italy); K. Schuster, J. Kobelke, K. Morl, IPHT Jena (Germany); S. Soria-Huguet, G. Nunzio Conti, G. C. Righini, Istituto di Fisica Applicata Nello Carrara, CNR (Italy) 
792508 Holographic spatiotemporal lens [7925-07]

K. Kimura, S. Hasegawa, Y. Hayasaki, Utsunomiya Univ. (Japan)

SESSION 3 OPTICAL PERFORATION OF CELLS BY FS LASERS I

7925 OB Towards high-throughput automated targeted femtosecond laser-based transfection of adherent cells [7925-10]

M. Antkowiak, M. L. Torres-Mapa, F. Gunn-Moore, K. Dholakia, Univ. of St. Andrews (United Kingdom)

\section{SESSION 4 OPTICAL PERFORATION OF CELLS BY FS LASERS II}

7925 OF Mechanisms of gold nanoparticle mediated ultrashort laser cell membrane perforation [7925-14]

M. Schomaker, Laser Zentrum Hannover e.V. (Germany); J. Baumgart, École Polytechnique de Montréal (Canada); D. Motekaitis, D. Heinemann, J. Krawinkel, Laser Zentrum Hannover e.V. (Germany); M. Pangalos, W. Bintig, Leibniz Univ. Hannover (Germany); E. Boulais, R. Lachaine, B. St.-Louis Lalonde, École Polytechnique de Montréal (Canada); A. Ngezahayo, Leibniz Univ. Hannover (Germany); M. Meunier, École Polytechnique de Montréal (Canada); A. Heisterkamp, Laser Zentrum Hannover e.V. (Germany)

7925 OG Basic mechanisms of the femtosecond laser interaction with a plasmonic nanostructure in water [7925-15]

E. Boulais, R. Lachaine, M. Meunier, École Polytechnique de Montréal (Canada)

7925 Ol Plasmonic enhanced fs-laser optoporation of human melanoma cells [7925-17] J. Baumgart, École Polytechnique de Montréal (Canada); L. Humbert, Royal Victoria Hospital (Canada); B. St.-Louis Lalonde, École Polytechnique de Montréal (Canada); J-J. Lebrun, Royal Victoria Hospital (Canada); M. Meunier, École Polytechnique de Montréal (Canada)

$79250 \mathrm{~J} \quad$ Gold nanoparticle mediated cell manipulation using fs and ps laser pulses for cell perforation and transfection [7925-18]

D. Heinemann, M. Schomaker, D. Motekaitis, J. Krawinkel, Laser Zentrum Hannover e.V. (Germany) and Excellence Cluster REBIRTH (Germany); D. Killian, Univ. of Rostock (Germany); H. M. Escobar, Univ. of Veterinary Medicine Hannover (Germany); C. Junghanß, Univ. of Rostock (Germany); A. Heisterkamp, Laser Zentrum Hannover e.V. (Germany) and Excellence Cluster REBIRTH (Germany)

SESSION $5 \quad$ FS LASER MODIFICATION OF TRANSPARENT MATERIALS I: STRUCTURING AND 3D MODIFICATION

7925 OK Direct laser written waveguide coupler with an optically tunable splitting ratio [7925-19] M. Ams, R. J. Williams, M. J. Withford, Macquarie Univ. (Australia) 
7925 OL Direct laser writing of nonlinear properties in photosensitive glass [7925-20]

G. Papon, Univ. Bordeaux 1 (France); J. Choi, College of Optics and Photonics, Univ. of Central Florida (United States) and Institut de Chimie de la Matière Condensée de Bordeaux, CNRS, Univ. Bordeaux 1 (France); M. Bellec, A. Royon, Univ. Bordeaux 1 (France); K. Bourhis, Institut de Chimie de la Matière Condensée de Bordeaux, CNRS, Univ. Bordeaux 1 (France); L. Canioni, Univ. Bordeaux 1 (France); T. Cardinal, Institut de Chimie de la Matière Condensée de Bordeaux, CNRS, Univ. Bordeaux 1 (France)

$79250 \mathrm{~N} \quad$ New step towards the future perennial high capacity optical recording medium [7925-22] A. Royon, K. Bourhis, G. Papon, M. Bellec, B. Bousquet, Y. Deshayes, T. Cardinal, L. Canioni, Univ. Bordeaux 1 (France)

\section{SESSION 6 FS LASER MODIFICATION OF TRANSPARENT MATERIALS II: MECHANISMS AND SIDE EFFECTS}

792500 Femtosecond filamentation induced micro- and nano-restructuring in the bulk of dielectrics and polymers (Invited Paper) [7925-23]

S. Tzortzakis, D. Abdollahpour, D. Papazoglou, S. Georgiou, Foundation for Research and Technology-Hellas (Greece)

7925 OP Breaking stress of glass welded with femtosecond laser pulses at high repetition rates (Best Paper Award) [7925-24]

S. Richter, S. Döring, Friedrich-Schiller-Univ. Jena (Germany); T. Peschel, R. Eberhardt, Fraunhofer Institute of Applied Optics and Precision Engineering (Germany); S. Nolte, A. Tünnermann, Friedrich-Schiller-Univ. Jena (Germany) and Fraunhofer Institute of Applied Optics and Precision Engineering (Germany)

$7925 \mathrm{OR}$ Time-resolved imaging of bulk a-SiO $\mathrm{S}_{2}$ upon various ultrashort excitation sequences [7925-26]

A. Mermillod-Blondin, Max-Born-Institut für Nichtlineare Optik und Kurzzeitspektroskopie (Germany); C. Mauclair, Max-Born-Institut für Nichtlineare Optik und Kurzzeitspektroskopie (Germany) and Lab. Hubert Curien, CNRS, Univ. de Lyon, Univ. Jean Monnet (France); A. Rosenfeld, Max-Born-Institut für Nichtlineare Optik und Kurzzeitspektroskopie (Germany); J. Bonse, Bundesanstalt für Materialforschung und -prüfung (Germany); R. Stoian,

E. Audouard, Lab. Hubert Curien, CNRS, Univ. de Lyon, Univ. Jean Monnet (France)

SESSION 7 LASER SOURCES AND SYSTEMS, MODELING, AND MEASUREMENT

7925 OS CPA-free picosecond fiber amplifier with $25 \mu \mathrm{J}$ pulse energy and $>500 \mathrm{~kW}$ peak power [7925-27]

Y. Zaouter, F. Morin, M. Lebugle, E. Mottay, C. Hönninger, Amplitude Systèmes (France)

7925 OT Technical advantages of disk laser technology in short and ultrashort pulse processes [7925-28]

P. Graham, J. Stollhof, TRUMPF Inc. (United States); S. Weiler, S. Massa, B. Faisst, TRUMPF Laser GmbH \& Co. KG (Germany); P. Denney, E. Gounaris, Connecticut Ctr. for Advanced Technology, Inc. (United States) 
7925 OU Versatile approach for frequency resolved wavefront characterization [7925-46]

E. Frumker, Joint Attosecond Science Lab. (Canada) and Texas A\&M Univ. (United States);

G. G. Paulus, Texas A\&M Univ. (United States) and Friedrich-Schiller-Univ. Jena (Germany);

H. Niikura, Joint Attosecond Science Lab. (Canada) and PRESTO JST (Japan);

D. M. Villeneuve, P. B. Corkum, Joint Attosecond Science Lab. (Canada)

$79250 \mathrm{~V}$ Optimal control of the population dynamics of the ground vibrational state of a polyatomic molecule [7925-30]

L. E. de Clercq, L. R. Botha, CSIR, National Laser Ctr. (South Africa) and Univ. of Stellenbosch (South Africa); E. G. Rohwer, CSIR, National Laser Ctr. (South Africa); H. Uys, Univ. of Stellenbosch (South Africa); A. Du Plessis, CSIR, National Laser Ctr. (South Africa) and Univ. of Stellenbosch (South Africa)

\section{SESSION 8 FS LASER MODIFICATION OF TRANSPARENT MATERIALS III}

$79250 X \quad$ Polymer micro-molding of femtosecond laser micromachined substrates [7925-47] M. Matteucci, F. Madani-Grasset, Y. Bellouard, Technische Univ. Eindhoven (Netherlands); D. Vitek, J. Squier, Colorado School of Mines (United States)

7925 OY Fs laser induced apodised Bragg waveguides in fused silica [7925-48] C. Voigtländer, Friedrich-Schiller-Univ. Jena (Germany); P. Zeil, Royal Institute of Technology (Sweden); J. Thomas, Friedrich-Schiller-Univ. Jena (Germany); M. Ams, R. J. Williams, M. J. Withford, Macquarie Univ. (Australia); A. Tünnermann, S. Nolte, Friedrich-Schiller-Univ. Jena (Germany) and Fraunhofer Institute for Applied Optics and Precision Engineering (Germany)

$79250 Z$ Role of stress in the chemical etching of fused silica exposed to low-energy femtosecond laser pulses [7925-49]

A. Champion, Y. Bellouard, Technische Univ. Eindhoven (Netherlands); G. Mindaugas,

M. Beresna, P. G. Kazansky, Univ. of Southampton (United Kingdom)

\section{SESSION 9 ULTRASHORT PULSE MICROMACHINING: JOINT SESSION WITH CONFERENCE 7920}

792510 Fundamentals and industrial applications of ultrashort pulsed lasers at Bosch (Invited Paper) [7925-32]

J. König, T. Baver, Robert Bosch GmbH (Germany)

792511 Directly induced ablation of metal thin films by ultrashort laser pulses [7925-33] G. Heise, J. Konrad, S. Sarrach, J. Sotrop, H. P. Huber, Munich Univ. of Applied Sciences (Germany)

792512 Colorizing of the stainless steel surface by single-beam direct femtosecond laser writing [7925-34]

M. S. Ahsan, Korea Advanced Institute of Science and Technology (Korea, Republic of) and Khulna Univ. (Bangladesh); Y. G. Kim, M. S. Lee, Korea Advanced Institute of Science and Technology (Korea, Republic of) 
792514 The effect of ambient conditions on thin wafers processed with fs-laser machining (Invited Paper) [7925-36]

S. C. Jeoung, Korea Research Institute of Standards and Science (Korea, Republic of)

792515 On the damage behaviour of $\mathrm{Al}_{2} \mathrm{O}_{3}$ insulating layers in thin film systems for the fabrication of sputtered strain gauges [7925-37]

O. Suttmann, U. Klug, R. Kling, Laser Zentrum Hannover e.V. (Germany)

SESSION 11 ONLINE MONITORING: JOINT SESSION WITH CONFERENCE 7920

792516 Inline coherent imaging of laser processing (Invited Paper) [7925-38]

J. M. Fraser, Queen's Univ. (Canada)

792517 In-situ observation of the hole formation during deep drilling with ultrashort laser pulses (Invited Paper) [7925-39]

S. Döring, S. Richter, Friedrich-Schiller-Univ. Jena (Germany); S. Nolte, A. Tünnermann, Friedrich-Schiller-Univ. Jena (Germany) and Fraunhofer Institute Applied Optics and Precision Engineering (Germany)

SESSION 12 FEMTOSECOND LASER NANOPROCESSING: JOINT SESSION WITH CONFERENCE 7920

792518 Ultrafast microsphere near-field nanostructuring [7925-40]

K.-H. Leitz, U. Quentin, B. Hornung, A. Otto, I. Alexeev, M. Schmidt, Univ. of ErlangenNuremburg (Germany)

\section{POSTER SESSION}

7925 1B Scattering-controlled femtosecond-laser induced nanostructuring of $\mathrm{TiO}_{2}$ thin films [7925-42] S. K. Das, A. Rosenfeld, M. Bock, Max-Born-Institut für Nichtlineare Optik und Kurzzeitspektroskopie (Germany); A. Pfuch, Friedrich-Schiller-Univ. Jena (Germany) and Innovent e.V. (Germany); W. Seeber, Friedrich-Schiller-Univ. Jena (Germany); R. Grunwald, Max-Born-Institut für Nichtlineare Optik und Kurzzeitspektroskopie (Germany)

7925 1C Invisible two-dimensional barcode fabrication inside a synthetic fused silica by femtosecond laser processing using a computer-generated hologram [7925-43] H. Kawashima, M. Yamaji, J. Suzuki, S. Tanaka, New Glass Forum (Japan)

7925 ID Optical device fabrication using femtosecond laser processing with glass-hologram [7925-44]

J. Suzuki, Y. Arima, S. Tanaka, New Glass Forum (Japan)

Author Index 
Downloaded From: https://www.spiedigitallibrary.org/conference-proceedings-of-spie on 26 Apr 2023

Terms of Use: https://www.spiedigitallibrary.org/terms-of-use 


\title{
Conference Committee
}

\author{
Symposium Chairs
}

Friedhelm Dorsch, TRUMPF GmbH \& Company KG (Germany)

Alberto Piqué, U.S. Naval Research Laboratory (United States)

Symposium Cochairs

Donald J. Harter, IMRA America, Inc. (United States)

Peter R. Herman, University of Toronto (Canada)

Conference Chairs

Alexander Heisterkamp, Laser Zentrum Hannover e.V. (Germany) Joseph Neev, Femto-Sec Tech, Inc. (United States)

Stefan Nolte, Friedrich-Schiller-Universität Jena (Germany)

Program Committee

Craig B. Arnold, Princeton University (United States)

James E. Carey III, SiOnyx Inc. (United States)

Xun Gu, Max-Planck-Institut für Quantenoptik (Germany)

Denise M. Krol, University of California, Davis (United States)

Holger Lubatschowski, Rowiak GmbH (Germany)

Eric D. Mazur, Harvard University (United States)

Michael M. Mielke, Raydiance, Inc. (United States)

Thomas E. Milner, The University of Texas at Austin (United States)

Eric Mottay, Amplitude Systèmes (France)

Christopher B. Schaffer, Cornell University (United States)

Alexander Szameit, Technion-Israel Institute of Technology (Israel)

Alfred Vogel, Universität zu Lübeck (Germany)

Wataru Watanabe, National Institute of Advanced Industrial Science and Technology (Japan)

\section{Session Chairs}

1 Biomedical Applications I: Imaging and Sensing Joseph Neev, Femto-Sec Tech, Inc. (United States)

2 Biomedical Applications II

Olivier Stachs, Universität Rostock (Germany) 
3 Optical Perforation of Cells by fs Lasers I

Alexander Heisterkamp, Laser Zentrum Hannover e.V. (Germany)

4 Optical Perforation of Cells by fs Lasers II

Christopher B. Schaffer, Cornell University (United States)

5 fs Laser Modification of Transparent Materials I: Structuring and 3D Modification

Stefan Nolte, Friedrich-Schiller-Universität Jena (Germany)

6 fs Laser Modification of Transparent Materials II: Mechanisms and Side Effects

Michael J. Withford, Macquarie University (Australia)

$7 \quad$ Laser Sources and Systems, Modeling, and Measurement Alexander Heisterkamp, Laser Zentrum Hannover e.V. (Germany)

8 fs Laser Modification of Transparent Materials III

Xun Gu, Max-Planck-Institut für Quantenoptik (Germany)

$9 \quad$ Ultrashort Pulse Micromachining: Joint Session with Conference 7920

Stefan Nolte, Friedrich-Schiller-Universität Jena (Germany

10 Thin Film and Wafer Processing: Joint Session with Conference 7920 Jens Koenig, Robert Bosch GmbH (Germany)

11 Online Monitoring: Joint Session with Conference 7920

Andreas Michalowski, Universität Stuttgart (Germany)

12 Femtosecond Laser Nanoprocessing: Joint Session with Conference 7920

Beat Neuenschwander, Berner Fachhochschule Technik und Informatik (Switzerland) 